\title{
BMJ Open Identifying indicators sensitive to primary healthcare nurse practitioner practice: a review of systematic reviews protocol
}

\author{
Kelley Kilpatrick (D) , ${ }^{1}$ Eric Nguemeleu Tchouaket (D) , ${ }^{2}$ Maud-Christine Chouinard, ${ }^{3}$ \\ Isabelle Savard, ${ }^{4}$ Naima Bouabdillah, ${ }^{5}$ Julie Houle, ${ }^{5}$ Geneviève St-Louis, ${ }^{6}$ \\ Mira Jabbour, ${ }^{7}$ Renee Atallah ${ }^{1}$
}

To cite: Kilpatrick K,

Tchouaket EN, Chouinard M-C, et al. Identifying indicators sensitive to primary healthcare nurse practitioner practice: a review of systematic reviews protocol. BMJ Open 2021;11:e043213. doi:10.1136/ bmjopen-2020-043213

- Prepublication history and additional material for this paper are available online. To view these files, please visit the journal online (http://dx.doi. org/10.1136/bmjopen-2020043213).

Received 27 July 2020 Revised 29 November 2020 Accepted 09 December 2020

Check for updates

(C) Author(s) (or their employer(s)) 2021. Re-use permitted under CC BY-NC. No commercial re-use. See rights and permissions. Published by BMJ.

For numbered affiliations see end of article.

Correspondence to Dr Kelley Kilpatrick; kelley.kilpatrick@mcgill.ca

\section{ABSTRACT}

Introduction Primary healthcare nurse practitioners (PHCNPs) practice in a wide range of clinical settings and with diverse patient populations. Several systematic reviews have examined outcomes of PHCNP roles. However, there is a lack of consistency in the definitions used for the PHCNP role across the reviews. The identification of indicators sensitive to PHCNP practice from the perspective of patients, providers and the healthcare system will allow researchers, clinicians and decision-makers to understand how these providers contribute to outcomes of care.

Methods and analysis A review of systematic reviews is proposed to describe the current state of knowledge about indicators sensitive to PHCNP practice using recognised role definitions. Outcomes of interest include any outcome indicator measuring the effectiveness of PHCNPs. We will limit our search to 2010 onwards to capture the most upto-date trends. The following electronic databases will be searched: Allied and Complementary Medicine Database, Cumulative Index to Nursing and Allied Health Literature, Cochrane Library Database of Systematic Reviews and Controlled Trials Register, Database of Abstracts of Reviews of Effects, EMBASE, Global Health, Health Economics Evaluation Database, Health Evidence, HealthStar, Health Systems Evidence, Joanna Briggs Institute, Medline, PDQ-Evidence, PubMed and Web of Science. The search strategies will be reviewed by an academic librarian. Reference lists of all relevant publications will be reviewed. Grey literature will be searched from 2010 onwards, and will include: CADTH Information Services, CADTH's Grey Matters tool, OpenGrey, Organisation for Economic Co-operation and Development, ProQuest Dissertation and Theses and WHO. The PROSPERO International Prospective Register of Systematic Reviews will be searched to identify registered review protocols. The review protocol was developed using Preferred Reporting Items for Systematic Review and MetaAnalysis Protocols recommendations. A narrative synthesis will be used to summarise study findings.

Ethics and dissemination No ethical approval is required for the study. The data used in the study will be abstracted from published systematic reviews. Dissemination strategies will include peer-reviewed publication, conference presentations and presentations to key stakeholders.
Strengths and limitations of this study

- Our review of systematic reviews will include a recognised definition for nurse practitioner (NP) roles, thereby helping bridge knowledge gaps resulting from the use of inconsistent role definitions that limit research finding generalisability across countries.

- There will be no language restrictions for studies included in the review of systematic reviews.

- No quantitative analyses are planned.

- Because the review focuses on studies that clearly define the NP role, the overview may exclude studies that examine NP roles but did not provide clear role definitions.

PROSPERO registration number CRD42020198182.

\section{BACKGROUND}

Internationally, the primary healthcare nurse practitioner (PHCNP) role has been examined across countries and healthcare settings (eg, long-term care, primary care, home care) to understand how PHCNPs provide care. ${ }^{1-4}$ PHCNPs are nurses prepared at the graduate level with in-depth clinical expertise who provide primary healthcare (PHC) services to different populations. ${ }^{5}$ Researchers ${ }^{6-9}$ have conducted several systematic reviews of PHCNP roles to understand their contributions to patient care. Laurant and colleagues completed a review of systematic reviews of the effectiveness of non-physician clinicians including PHCNPs. ${ }^{10}$ These researchers identified 18 systematic reviews but noted that an 'exact description of the nurses' roles was lacking in the majority of reviews' ${ }^{10,}$ p. ${ }^{44 S}$ Kilpatrick et al conducted a review of reviews to examine the impact of graduate-prepared nurses (nurse practitioners (NPs) and clinical nurse specialists) regardless of their clinical practice setting. ${ }^{11}$ This review of reviews 
identified 4 systematic reviews examining 24 outcomes across reviews including 18 patient, 1 provider and 5 healthcare system outcomes for the 2 advanced practice nursing roles in acute and primary care ${ }^{11}$ Internationally, system-level dimensions have consistently been identified in the delivery of safe, efficient and effective PHC, ${ }^{12-14}$ among them, access, comprehensiveness, continuity of care, coordination of care, equity, integration of care and patient-centredness. A recent review of systematic reviews of PHC quality indicators identified 727 quality indicators where almost $75 \%$ of the indicators focused on process indicators (eg, treatment). ${ }^{15}$ This umbrella review highlighted that subsequent reviews needed to identify the measurement characteristics of quality indicators (eg, denominator and calculation method of a quality indicator) to capture the contributions of PHC providers. In addition, workforce data to measure the contributions of non-physician providers including PCHNPs are needed globally. ${ }^{16}{ }^{17}$ Internationally, PHCNP roles are expanding into new areas of practice to support the delivery of patient-centred PHC. An important gap remains in identifying indicators that have been used to document the contributions of PHCNPs across settings. Thus, to understand PHCNP contributions to patient care and synthesise the available evidence, we propose to build on previous research by conducting a review of systematic reviews of studies that incorporates a recognised PHCNP role definition to identify indicators sensitive to PHCNP practice. These findings will inform the evaluation of PHCNP practice. Our research question is: what indicators are sensitive to the practice of PHCNPs from the patient, provider and health system perspectives?

In this paper, we propose to outline the methodology used in the review of systematic reviews. We aim to evaluate and synthesise systematic reviews to identify outcome indicators sensitive to the practice of PHCNPs from the patient, provider and health system perspectives. More specifically, our objectives are:

\section{Objective 1}

To assess the quality of systematic reviews of the impact of PHCNP practice from the patient, provider and health system perspectives.

\section{Objective 2}

To identify indicators sensitive to the practice of PHCNPs from the patient, provider and health system perspectives.

\section{METHODS}

We will conduct an overview of systematic reviews to describe the current state of knowledge about indicators sensitive to PHCNP practice from the patient, provider and health system perspectives. ${ }^{18}$ The review protocol was developed using Preferred Reporting Items for Systematic Review and Meta-Analysis Protocols recommendations of Shamseer et al. ${ }^{19}$

\section{Inclusion criteria}

Types of studies

All relevant published and unpublished systematic reviews that were reported from 2010 and onwards with no restrictions on jurisdiction or language. To be identified as a systematic review, the authors need to identify a specific research question or provide sufficient information so the reviewer can identify all the components of a research question (ie, Participants, Intervention, Comparison, Outcomes, Study Design (PICOS)) related to indicators sensitive to PHCNP practice, prespecify inclusion and exclusion criteria, and use systematic methods to identify relevant published and unpublished evidence to minimise the risk of bias. ${ }^{20}$ The review will be included provided their inclusion criteria required the PHCNP role to be clearly defined.

\section{Designs in included studies}

Jackson and Waters ${ }^{21}$ argue that understanding the impact of complex interventions like the addition of a PHCNP role in healthcare teams necessitates different types of information to inform decisions about their effectiveness. Systematic reviews included in our overview may include the results of randomised controlled trials, prospective controlled observational studies and cohort studies, retrospective controlled observational and cohort studies, and surveys. A summary table will be developed to present key findings.

\section{Types of participants}

Participants include patients of any age, groups or communities receiving PHC care in all types (eg, teaching and non-teaching, public and private), sizes (eg, small, medium, large) and locations (eg, rural and urban) of community or care agencies (eg, long-term care, primary care practices, home care). Data will be extracted to describe the total number of participants, specific health conditions (eg, diabetes, hypertension) or type of care (eg, well baby care).

\section{Types of interventions}

We will include reviews that examine the care provided by PHCNPs in all sectors. We will adopt a recognised definition of the PHCNP role. PHCNPs are registered nurses with additional educational preparation at the master's level or above and in-depth clinical expertise who possess and demonstrate the competencies to autonomously diagnose, order and interpret diagnostic tests, prescribe pharmaceuticals and perform specific procedures within their legislated scope of practice. ${ }^{22}$ To be considered an $\mathrm{NP}$, the review has to specify that the nurse has completed a formal post-baccalaureate or graduate NP education programme.

We will determine whether the PHCNPs are working in complementary, alternative, transition or mixed roles. According to Laurant and colleagues,${ }^{10}$ those working predominantly in complementary roles provide additional services that are intended to complement or extend 
existing services, and those working in predominantly alternative roles provide similar services to those for whom they are substituting (usually physicians). Those working in transition roles facilitate the safe and timely transfer of patients from one care site to another (eg, hospital to home or to a skilled nursing facility) or from one level of care to another. ${ }^{23}$ Some PHCNPs provide services that are a combination of complementary and alternative roles. These will be categorised as mixed roles.

\section{Types of comparators}

Data related to the comparator (ie, control) group will be extracted to describe the group to which care is compared. Comparator groups can include usual care, care provided by another healthcare professional (eg, physicians), best care or adherence to clinical practice guideline. Data extractors will extract a brief description of the control group.

\section{Types of outcomes}

Outcomes of interest in this review of reviews include any outcome indicator that measures the effectiveness of PHCNPs. These measures include patient (eg, health status, quality of life, patient satisfaction) or provider (eg, quality of care, job satisfaction) or health system (eg, length of hospital stay, rehospitalisation, resource use, costs) outcomes. We will extract data related to effect sizes, and include the actual effect size (ie, OR, relative risk, mean differences), CIs, level of statistical significance and the number of studies included in the analysis. ${ }^{10}$

\section{Exclusion criteria}

Reviews that were developed to address broad research questions (eg, literature reviews, scoping reviews, integrative reviews) will be excluded.

Nurse midwives will be excluded from the review of reviews because the regulatory requirements in different countries do not all require these roles to be filled by nurses, and nurse midwife roles are not consistently identified as advanced practice nursing roles across countries.

Reviews will be excluded if PHCNP impact cannot be isolated and is not reported separately from other types of nurses or team members. A list of all excluded reviews will be developed specifying reasons for exclusion.

\section{Database search}

Evidence in approximately half of published reviews is outdated after 5 years. ${ }^{24}$ Thus, we will limit our search to 2010 onwards to capture the most up-to-date trends. The following electronic databases will be searched: Allied and Complementary Medicine Database, Cumulative Index to Nursing and Allied Health Literature, Cochrane Library Database of Systematic Reviews and Controlled Trials Register, Database of Abstracts of Reviews of Effects, EMBASE, Global Health, Health Economics Evaluation Database, Health Evidence, HealthStar, Heath Systems Evidence, Joanna Briggs Institute, Medline, PDQEvidence, PubMed and Web of Science. The preliminary search strategies to be adapted for each electronic database are included in online supplemental appendix 1. These strategies will be reviewed by an academic librarian. In addition, the reference lists of all relevant reviews will be reviewed.

The grey literature will be searched for the period of 2010 and onwards, and will include: Canadian Agency for Drugs and Technologies in Health (CADTH) Information Services, relevant websites from CADTH's Grey Matters tool, OpenGrey Repository, Organisation for Economic Co-operation and Development, ProQuest Dissertation and Theses and WHO (online supplemental appendix 2). The PROSPERO International Prospective Register of Systematic Reviews will be searched to identify registered review protocols. Authors of registered PROSPERO reviews will be contacted to ascertain the status of the study. For each website, the content will be searched using the same search terms as those used for the published literature. If there is not an inherent search function on the website, a search will be conducted of all webpages and weblinks. The preliminary search strategy for the unpublished literature is included in online supplemental appendix 2.

\section{Study selection}

All reviewers will be trained to use the screening instrument and the inclusion/exclusion criteria. The retained studies will be uploaded into the EndNote software and the RAYYAN web platform. ${ }^{25}$ Duplicates will be removed. Two reviewers will independently screen titles and abstracts using the predefined inclusion and exclusion criteria, and recommend exclusion or further full text review. Any discrepancies will be discussed among the reviewers. If there is insufficient information in the abstract or no abstract is available, a full text review will be completed. Any coding discrepancies will be discussed between the reviewers until agreement is reached on the inclusion or exclusion of the review. A third reviewer will act as a tie-breaker if the first reviewers do not come to a consensus. A full text review will be completed for all the reviews included after the initial screening again using the predefined inclusion and exclusion criteria.

\section{Data extraction}

For those full text papers that pass inclusion criteria, the data will be extracted by one coder and reviewed by a second coder. A structured tool will be developed for the study and pilot-tested by the investigators. ${ }^{26}$ We will extract data from the methods and results section. The extracted data will include aim or focus of the review; review characteristics (eg, year); number and name of electronic databases searched; characteristics of the participants and intervention (ie, complementary, alternative, transition or mixed roles); number and types of studies included in the review; specification of patient, provider and health system outcomes and how the outcomes are measured; risk of bias assessment tool used; the quality ratings of the included studies and funding source. ${ }^{27} 28$ The results of the meta-analyses, if conducted, will be included in the 
data extraction. If the data are not available in the review they will be identified as 'unknown' in the data extraction form.

\section{Assessment of review quality}

Each review will be graded using the AMSTAR 2 criteria to assess its methodological quality. Two reviewers will independently rate each review using the AMSTAR 2 criteria. $^{29}$ Inter-rater agreement will be estimated using the kappa statistic. ${ }^{30}$ Any disagreements will be discussed among the reviewers until consensus is reached. A summary table with the AMSTAR ratings will be generated.

\section{Outcomes}

The primary outcomes of the review of reviews are those that assess patient, provider and health system outcomes directly evaluating PHCNP roles.

These will be examined separately for type of PHCNP role (ie, complementary, alternative, transition, mixed).

\section{Data synthesis}

Narrative synthesis will be used to summarise the findings. As proposed by Olry de Labry Lima et al and Ramalho et $a l$, outcomes of care provided by PHCNPs will be categorised according to clinical indicators of care quality (eg, glycated haemoglobin for patients with diabetes), medications prescribed for chronic conditions (process), health promotion (eg, healthy lifestyle) and illness prevention activities (eg, influenza vaccination). ${ }^{15} 31$ An iterative process will be used to identify patterns and relationships across reviews and across years that the reviews were conducted.$^{32}$ Summary tables will be developed to outline the key characteristics of the reviews (eg, year of publication, countries where primary studies were conducted), outcomes (ie, patient, provider, health system), level of role substitution (ie, complementary, alternative, transition, mixed), quality assessment and findings (positive, negative, null). A record will be kept of all review-related decisions. No quantitative analyses are planned because of the potential overlap in studies included in the different reviews. ${ }^{18}$

\section{Patient and public involvement}

This review of systematic reviews was undertaken without patient involvement. Patients were not invited to comment on the study protocol design. Patients were not invited to contribute to the writing nor the editing of this manuscript. An expert patient will be consulted as to how this work may inform patient relevant outcomes or how a patient might interpret results. The results of this work will be disseminated to key stakeholders including decision makers, healthcare professionals and patients via conferences, publications and presentations.

\section{Anticipated contributions}

PHCNPs practice in a wide range of settings and with diverse patient populations, including vulnerable populations. ${ }^{33}$ The identification of indicators sensitive to the practice of PHCNPs from the perspective of patients, providers and the healthcare system will allow researchers, clinicians and decision-makers to understand how these providers contribute to outcomes of care. Gaining an understanding of the patient perspective is particularly important in the context of patient-centred care and adapting services to the needs of vulnerable populations (eg, residents in long-term care, patients with mental health conditions or low socioeconomic status). Once identified, PHCNPs, other clinicians and decision-makers can track these indicators and determine if PHCNP roles are used optimally to respond to patient care needs. Using these indicators, subsequent research can be undertaken to support more rigorous economic evaluations of these roles, an important gap in the current literature. ${ }^{34} 35$

\section{Ethics and dissemination}

No ethical approval is required for the study. The data used in the study will be abstracted from published systematic reviews. Dissemination strategies will include peer-reviewed publication, conference presentations and presentations to key stakeholders.

\section{Author affiliations}

${ }^{1}$ Susan E. French Chair in Nursing Research and Innovative Practice, Ingram School of Nursing, Faculty of Medicine, McGill University, Montréal, Québec, Canada

${ }^{2}$ Département des sciences infirmières, Université du Québec en Outaouais, Saint Jérôme, Québec, Canada

${ }^{3}$ Département des sciences de la santé (Health Sciences Department), Université du Québec à Chicoutimi, Chicoutimi, Québec, Canada

${ }^{4}$ Ingram School of Nursing, Faculty of Medicine and Health Sciences, McGill University, Montreal, Quebec, Canada

${ }^{5}$ Département des sciences infirmières, Université du Québec à Trois-Rivières, TroisRivières, Québec, Canada

${ }^{6}$ Support and Development of Professional Practices in Nursing and Assistance Care and Infection Prevention Associate Directorate, Centre intégré universitaire de santé et de services sociaux de la Mauricie-et-du-Centre-du-Québec, Trois-Rivieres, Quebec, Canada

${ }^{7}$ Maisonneuve-Rosemont Hospital Site, Centre intégré universitaire de santé et de services sociaux de l'Est-de-I'Île-de-Montréal du Québec, Montreal, Quebec, Canada

Correction notice This article has been corrected since it first published. The provenance and peer review statement has been included.

Twitter Maud-Christine Chouinard @MaudCChouinard

Acknowledgements This study was funded by the Le Réseau intersectoriel de recherche en santé de l'Université du Québec (RISUQ) (Project Grant Number 304168 (30416801); principal investigator: ENT). KK and ENT are supported by the Fonds de recherche du Québec-Santé (Research Scholar Junior 2 (KK) (Award Number 34722) and Junior 1 (ENT) (Award Number 31051) salary awards.

Contributors KK, ENT, M-CC, IS, NB, JH, GS-L, MJ and RA made substantial contributions to study conception and design. All authors were involved in drafting the manuscript and making revisions for critical intellectual content. All authors gave final approval of the version to be published.

Funding Le Réseau intersectoriel de recherche en santé de l'Université du Québec (RISUQ) (Project Grant Number: 304168 (30416801); principal investigator: ENT). The funder played no role in: study design and interpretation; development of the manuscript.

Competing interests None declared.

Patient consent for publication Not required.

Provenance and peer review Not commissioned; externally peer reviewed.

Supplemental material This content has been supplied by the author(s). It has not been vetted by BMJ Publishing Group Limited (BMJ) and may not have been peer-reviewed. Any opinions or recommendations discussed are solely those 
of the author(s) and are not endorsed by BMJ. BMJ disclaims all liability and responsibility arising from any reliance placed on the content. Where the content includes any translated material, BMJ does not warrant the accuracy and reliability of the translations (including but not limited to local regulations, clinical guidelines, terminology, drug names and drug dosages), and is not responsible for any error and/or omissions arising from translation and adaptation or otherwise.

Open access This is an open access article distributed in accordance with the Creative Commons Attribution Non Commercial (CC BY-NC 4.0) license, which permits others to distribute, remix, adapt, build upon this work non-commercially, and license their derivative works on different terms, provided the original work is properly cited, appropriate credit is given, any changes made indicated, and the use is non-commercial. See: http://creativecommons.org/licenses/by-nc/4.0/.

\section{ORCID iDs}

Kelley Kilpatrick http://orcid.org/0000-0003-2137-6560

Eric Nguemeleu Tchouaket http://orcid.org/0000-0002-4309-0478

\section{REFERENCES}

1 Chavez KS, Dwyer AA, Ramelet A-S. International practice settings, interventions and outcomes of nurse practitioners in geriatric care: a scoping review. Int J Nurs Stud 2018;78:61-75.

2 Kilpatrick K, Tchouaket $\mathrm{E}$, Jabbour $\mathrm{M}$, et al. A mixed methods quality improvement study to implement nurse practitioner roles and improve care for residents in long-term care facilities. BMC Nurs 2020;19:1-14.

3 Grant J, Lines L, Darbyshire P, et al. How do nurse practitioners work in primary health care settings? A scoping review. Int J Nurs Stud 2017;75:51-7.

4 Martin-Misener R, Donald F, Wickson-Griffiths A, et al. A mixed methods study of the work patterns of full-time nurse practitioners in nursing homes. J Clin Nurs 2015;24:1327-37.

5 Landry V, Kilpatrick K, Paquette L, et al. L'étendue du rôle de l'infirmière praticienne spécialisée en soins de première ligne dans différents milieux de pratique au Québec: une étude temps et mouvement. SNAHP 2020;3:1-14.

6 Martin-Misener R, Harbman P, Donald F, et al. Cost-Effectiveness of nurse practitioners in primary and specialised ambulatory care: systematic review. BMJ Open 2015;5:e007167.

7 Newhouse RP, Stanik-Hutt J, White KM, et al. Advanced practice nurse outcomes 1990-2008: a systematic review. Nurs Econ 2011;29:230-50.

8 Swan M, Ferguson S, Chang A, et al. Quality of primary care by advanced practice nurses: a systematic review. Int J Qual Health Care 2015;27:396-404.

9 Donald F, Kilpatrick K, Reid K, et al. A systematic review of the costeffectiveness of nurse practitioners and clinical nurse specialists: what is the quality of the evidence? Nurs Res Pract 2014;2014:1-28.

10 Laurant M, Harmsen M, Wollersheim H, et al. The impact of nonphysician clinicians: do they improve the quality and costeffectiveness of health care services? Med Care Res Rev 2009;66:36S-89.

11 Kilpatrick K, DiCenso A, Reid K. The impact of Graduate-Prepared nurse practitioners and clinical nurse specialists on patient, provider and health system outcomes: a review of systematic reviews. 9th biennial Joanna Briggs International Colloquium, scaling new heights: challenging the status quo. Singapore, 2014.

12 Haj-Ali W, Hutchison B. Establishing a primary care performance measurement framework for Ontario. Mise en place d'un cadre pour la mesure du rendement des soins primaires en Ontario. Healthc Policy 2017;12:66-79.

13 Kringos DS, Boerma WGW, Hutchinson A, et al. The breadth of primary care: a systematic literature review of its core dimensions. BMC Health Serv Res 2010;10:1-13.

14 Ontario HQ. Quality in primary care: setting foundation for monitoring and reporting in Ontario, 2015. Available: www.hqontario.ca/Portals/
0/Documents/pr/theme-report-quality-in-primary-care-en.pdf [Accessed 22 Oct 2020].

15 Ramalho A, Castro P, Gonçalves-Pinho M, et al. Primary health care quality indicators: an umbrella review. PLoS One 2019;14:e0220888.

16 Ammi M, Ambrose S, Hogg B, et al. The influence of registered nurses and nurse practitioners on patient experience with primary care: results from the Canadian QUALICO-PC study. Health Policy 2017;121:1215-24.

17 World Health Organization. State of the World's Nursing Report 2020- Investing in education, jobs and leadership. Health Workforce 6 April 2020. Available: https://www.who.int/publications/i/item/ 9789240003279 [Accessed 22 Oct 2020].

18 Smith V, Devane D, Begley CM, et al. Methodology in conducting a systematic review of systematic reviews of healthcare interventions. BMC Med Res Methodol 2011;11:15.

19 Shamseer L, Moher D, Clarke M, et al. Preferred reporting items for systematic review and meta-analysis protocols (PRISMA-P) 2015: elaboration and explanation. BMJ 2015;350:g7647.

20 Liberati A, Altman DG, Tetzlaff J, et al. The PRISMA statement for reporting systematic reviews and meta-analyses of studies that evaluate health care interventions: explanation and elaboration. PLoS Med 2009;6:e1000100.

21 Jackson N, Waters E. Criteria for the guidelines for systematic reviews in health promotion and public health Taskforce. Health Promot Int 2005;20:367-74.

22 Bowen D, Bryant Lukosius D, Byres D. Advanced practice nursing: A Pan-Canadian framework. CAN, 2019. Available: https://www. cna-aiic.ca/-/media/cna/page-content/pdf-en/apn-a-pan-canadianframework.pdf?la=en\&hash=E1387634D492FD2B003964E3CD41 88971305469E [Accessed 22 Oct 2020].

23 Naylor MD, Aiken LH, Kurtzman ET, et al. The care span: the importance of transitional care in achieving health reform. Health Aff 2011;30:746-54.

24 Pieper D, Buechter R, Jerinic P, et al. Overviews of reviews often have limited rigor: a systematic review. J Clin Epidemiol 2012;65:1267-73.

25 Ouzzani M, Hammady H, Fedorowicz Z, et al. Rayyan-a web and mobile APP for systematic reviews. Syst Rev 2016;5:210.

26 Kilpatrick K, Paquette L, Jabbour M, et al. Systematic review of the characteristics of brief team interventions to clarify roles and improve functioning in healthcare teams. PLoS One 2020;15:e0234416.

27 Hart LC, Patel-Nguyen SV, Merkley MG, et al. An evidence map for interventions addressing transition from pediatric to adult care: a systematic review of systematic reviews. J Pediatr Nurs 2019;48:18-34

28 Aromataris E, Fernandez R, Godfrey CM, et al. Summarizing systematic reviews: methodological development, conduct and reporting of an umbrella review approach. Int J Evid Based Healthc 2015;13:132-40.

29 Shea BJ, Reeves BC, Wells G, et al. AMSTAR 2: a critical appraisal tool for systematic reviews that include randomised or nonrandomised studies of healthcare interventions, or both. BMJ 2017;358:j4008.

$30 \mathrm{Sim}$ J, Wright CC. The kappa statistic in reliability studies: use, interpretation, and sample size requirements. Phys Ther 2005;85:257-68.

31 Olry de Labry Lima A, García Mochón L, Bermúdez Tamayo C. Identification of health outcome indicators in Primary Care. A review of systematic reviews. Rev Calid Asist 2017;32:278-88.

32 Whittemore R, Knafl K. The integrative review: updated methodology. $J$ Adv Nurs 2005;52:546-53.

$33 \mathrm{Xue} \mathrm{Y}$, Intrator $\mathrm{O}$. Cultivating the role of nurse practitioners in providing primary care to vulnerable populations in an era of healthcare reform. Policy Polit Nurs Pract 2016;17:24-31.

34 Tchouaket E, Kilpatrick K, Jabbour M. Effectiveness of introducing nurse practitioners in six long-term care facilities in Québec, Canada: a cost-savings analysis. Nurs Outlook 2020;6554:30562-7.

35 Marshall DA, Donald F, Lacny S, et al. Assessing the quality of economic evaluations of clinical nurse specialists and nurse practitioners: a systematic review of cost-effectiveness. NursingPlus Open 2015;1:11-17. 